\title{
Astrometry of satellites I to V of Uranus 1990-1991*
}

\author{
D.H.P. Jones ${ }^{1,2}$, D.B. Taylor ${ }^{1}$, and I.P. Williams ${ }^{2}$ \\ 1 Royal Greenwich Observatory, Madingley Rd., Cambridge, CB3 0EZ, UK \\ 2 Astronomy Unit, School of Mathematical Sciences, Queen Mary and Westfield College, Mile End Road, London E1 4NS, UK
}

Received September 25; accepted November 7, 1997

\begin{abstract}
CCD images of the Uranian satellite system taken with the Jacobus Kapteyn Telescope in 1990 and 1991 have been calibrated astrometrically with two WFC photographic plates taken on the same telescope in 1996. The numbers of images are: I Ariel 139; II Umbriel 132; III Titania 137; IV Oberon 128; V Miranda 117; total 653. The root-mean-square (rms) fits of the inter-satellite positions to the numerical integration by Taylor (1998) lie between 0 .'025 and $00^{\prime \prime} 061$, depending on which two satellites are compared.
\end{abstract}

Key words: planets and satellites: satellites of Uranus observations astrometry

\section{Introduction}

Satellites I - V of Uranus have orbits closely aligned to the planet's equatorial plane. However unlike any other planet in the Solar System the equator of Uranus has an inclination near $90^{\circ}$ whereas the rest are closer to zero. The satellite orbits were widest open in 1986 and are edgeon 21 years before and after. The eccentricities are best determined while the orbits are open and the inclinations when seen edge-on.

Although the surfaces of Uranus and his satellites can be studied from space, their interior structure can be studied only from their dynamical interaction. In principle their mutual perturbations should reveal their masses and the dynamical compression of the planet $J_{2}$; but Taylor (1998) has shown that it is not possible to determine the compression and the masses of the five largest satellites simultaneously. This indeterminacy is not fundamental but arises from the limitations of the dataset, already confined to only the best modern observations. The objective of the

Send offprint requests to: D.H.P. Jones, dhpj@ast.cam.ac.uk

* Table 7 is only available in electronic form at the CDS via anonymous ftp to cdsarc.u-strasbg.fr (130.79.128.5) or via http://cdsweb.u-strasbg.fr/Abstract.html present investigation is to add to the dataset in both size and accuracy with a view to removing this indeterminacy. A preliminary reduction of these observations was used by Taylor (1998); those presented here have been reduced with the more elaborate procedure described below.

At the 1990 and 1991 oppositions we observed Uranus with his satellites through a Gunn $Z$ filter (effective wavelength $\sim 0.9 \mu$ ) with a GEC CCD. The field of the chip was $3^{\prime} \times 2^{\prime}$. Contemporaneous observations of the satellites of Saturn have been published by Harper et al. (1997) with the same instrumental set-up. Harper et al. had insufficient data to calibrate the scale and orientation of the chip independently, and so resorted to calibrating with the well-known orbits of the brighter satellites.

\section{Photographic observations}

The orientation and scale of the CCD chip can easily be measured if there are sufficient stars of accurately known position on the same CCD images. To measure star positions in the area of sky crossed by Uranus during 1990 and 1991 we exposed two photographic plates with the Wide Field Camera (WFC) on the Jacobus Kapteyn Telescope (JKT); $28^{\circ} \quad 45^{\prime} \quad 39^{\prime \prime} \cdot 9 \quad \mathrm{~N} \quad 17^{\circ} \quad 52^{\prime} \quad 41^{\prime \prime} .2 \quad \mathrm{~W} \quad 2364 \mathrm{~m}$. The plates (Table 1) were exposed at the $f / 8.06$ HarmerWynne focus where the plate scale is $25^{\prime \prime} 67 / \mathrm{mm}$ and the field diameter 1.5, Harmer \& Wynne (1977), Carter et al. (1995).

Table 1. Details of photographic plates

\begin{tabular}{llll}
\hline Plate Date & \multicolumn{4}{c}{ UT Mid Exp. RA(J2000.0) } & Dec(J2000.0) \\
\hline 1028 & 1996 May $142^{\mathrm{h}} 17^{\mathrm{m}} 16^{\mathrm{s}}$ & $18^{\mathrm{h}} 30^{\mathrm{m}} 04^{\mathrm{s}} .9$ & $-23^{\circ} 35^{\prime} 22^{\prime \prime}$ \\
1029 & 1996 May $142^{\mathrm{h}} 56^{\mathrm{m}} 40^{\mathrm{s}}$ & $18^{\mathrm{h}} 51^{\mathrm{m}} 55^{\mathrm{s}} .9$ & $-23^{\circ} 16^{\prime} 51^{\prime \prime}$ \\
\hline
\end{tabular}

For both plates the emulsion was Kodak IIIaF hypersensitised by baking in Forming Gas for 2 hours at 
$65{ }^{\circ} \mathrm{C}$, the filter $4 \mathrm{~mm}$ RG630, and the exposure $32 \mathrm{mins}$. This reproduces the $R$ band with effective wavelength $650 \mathrm{~nm}$. The $R$ band was chosen to minimise atmospheric dispersion as the plates were exposed at a zenith distance $\sim 50^{\circ}$. Experience gained from similar plates suggests that the faintest images of astrometric quality are $R \approx 17$.

The plates were measured on the RGO PDS in Cambridge at orientations of $0^{\circ}$ and $90^{\circ}$. The reference stars which were taken from the PPM catalogue (Bastian \& Röser 1993) and the measures were reduced with a six coefficient fit after removing the distortion of the Harmer-Wynne design following Taylor et al. (1990). The ASTROM program written by Wallace (1994) was used for all reductions.

The 16 reference stars were measured at the beginning and end of both measuring runs so that there are four measures of each; thus the rms error of the measuring machine and the rms error of the catalogue can be separated and are presented in Table 2.

Table 2. Photographic measuring errors

\begin{tabular}{ll}
\hline Source of Error & Amount \\
\hline Measuring error of one image in one co-ordinate & $0^{\prime \prime} 04$ \\
Rms error of PPM at 1996.4 in one co-ordinate & 0.15 \\
Expected rms error of these 16 PPM stars & 0.12 \\
\hline
\end{tabular}

The measuring error is the accuracy with which the machine can centre on an image. The rms error of the PPM is the goodness of fit of the measures to the PPM positions. The expected error is derived from the published errors in the PPM Catalogue. The last two values are expected to be the same; their difference is not statistically significant for samples of this size.

The plates were compared with the CCD images and 166 stars in common were identified and measured. As each programme star was measured twice the expected internal rms error in one co-ordinate is $00^{\prime \prime} 03$. There are only eight stars in common with the GSC1.2 (Morrison \& McClean 1996).

\section{CCD observations}

66 CCD images were exposed between 1990 July 10 and 18, two on 1990 October 21, and 71 between 1991 July 4 and 11 . The $Z$ filter $(\lambda \sim 0.9 \mu)$ was chosen primarily because the large amounts of methane in the atmosphere of Uranus make the planet faint at this wavelength, while the satellites are relatively unaffected. Further advantages are that, compared to other broad-band colours available to the CCD:- (1) moonlight on the image arises mostly by
Rayleigh scattering which is smallest at this wavelength. Many of these observations were made with the full Moon close to the field. (2) the small atmospheric dispersion at this wavelength.

All the CCD reductions were done with IRAF. The CCD images were bias-subtracted, and flat-fielded with images of the twilight sky. The co-ordinates of the satellites and the secondary astrometric stars were found from PHOT, PSTSELECT, PSF and PEAK. PSTSELECT was used to identify the four brightest unblended stars from the list provided by PHOT. PSF derived the point-speadfunction (psf), consisting of independent Gaussian distributions in $x$ and $y$ from these four stars. PEAK fitted the centres of all the images with the psf.

Miranda is more or less blended with Uranus depending on the seeing, although the use of the $Z$ filter minimises the effect. Other observers working at shorter wavelengths have removed the blending by modelling and then subtracting the light of Uranus before centring on Miranda (Vieira Martins et al. 1986; Pascu et al. 1987; Veiga \& Vieira Martins 1995a). Pascu et al. also cut down the light of Uranus with a coronagraph and Veiga and Vieira Martins simply rotated the image of Uranus through $180^{\circ}$ before subtraction.

We removed the variable background by subtracting an image smoothed with a ring filter following Secker (1995). From each CCD image we subtracted an image which had been smoothed with IRAF FRMEDIAN. The ring had a radius twice the full-width-at-half-maximum (FWHM) of the seeing disc and width one pixel.

During these observations Uranus was moving through rich star fields towards the centre of the Milky Way and on several occasions the satellites were blended with stars. Misshapen images could be identified from the value of "chi" in PEAK. All satellite images with chi $>2.5$ were rejected. Another symptom of blending is that the satellite appears abnormally bright. Table 3 gives the median and semi-inter-quartile (siq) $Z$ magnitudes of the satellites from all our images; relative to Titania, the brightest. Any satellite which deviated by more than seven times the siq was rejected. Table 3 follows the IAU numbering system and also includes the $(V-Z)$ colours formed by differencing these magnitudes with those given by Reitsema et al. (1978).

Table 3. Median magnitudes relative to Titania III

\begin{tabular}{lllr}
\hline Satellite & Median & Siq & $V-Z$ \\
\hline Ariel I & 0.41 & 0.03 & -0.20 \\
Umbriel II & 1.05 & 0.03 & -0.01 \\
Oberon IV & 0.22 & 0.02 & -0.07 \\
Miranda V & 2.52 & 0.06 & 0.00 \\
\hline
\end{tabular}


The images were reduced with ASTROM (Wallace 1994) to give the scale and orientation of each. Any proper motion of the stars between the epoch of the plates and the epoch of the CCD observations has been neglected. The goodness of fit in ASTROM is a combination of errors in the reference star positions and errors in extracting the image centres from the CCD. A well observed night was 1990 July 13/14 when 15 images were obtained with 12 reference stars common to all. For this and similar nights it is straightforward to separate the errors (Table 4).

Table 4. Rms errors of CCD observations

\begin{tabular}{lll}
\hline Source & RA & Dec \\
\hline CCD error & $0^{\prime \prime} 012$ & $0^{\prime \prime} 012$ \\
Star Positions & $0^{\prime \prime} 039$ & $0^{\prime \prime} 031$ \\
Combined & $0^{\prime \prime} 041$ & $0^{\prime \prime} 033$ \\
\hline
\end{tabular}

The star position errors roughly agree with the photographic measuring errors in Table 2. The CCD errors are 0.04 pixel which is three times the value found by Zacharias (1996). However, Zacharias made contiguous exposures with the telescope autoguided where our exposures are spread over five hours and without autoguiding.

\section{Comparison of scale and position angle with Harper et al. (1997)}

The observations made by Harper et al. of the satellites of Saturn were made with exactly the same instrumentation and should show the same scale and orientation. However Harper et al. made their calibration by fitting to the wellknown orbits of the brighter satellites whereas the present calibration is based on star positions. The two are compared in Table 5 where the orientation is relative to the true pole of date. The discrepancies between Harper et al. and the present work in Table 5 are slightly greater than expected and this doubtless arises from the calibration procedures.

Table 5. Calibration parameters for the CCD observations

\begin{tabular}{lll}
\hline Dataset & Orientation & scale $(\operatorname{arcsec} /$ pixel $)$ \\
\hline Harper et al. (1990) & $1.1746 \pm 0.0030$ & $0^{\prime \prime} 302844 \pm 0^{\prime \prime} 000016$ \\
This paper (1990) & $1.1883 \pm 0.0026$ & $0^{\prime \prime} 302752 \pm 0^{\prime \prime} 000027$ \\
Harper et al. (1991) & $0.2682 \pm 0.0050$ & $0^{\prime \prime} 302936 \pm 0^{\prime \prime} 000027$ \\
This paper (1991) & $0.2675 \pm 0.0046$ & $0^{\prime \prime} 302777 \pm 0^{\prime \prime} .000030$ \\
\hline
\end{tabular}

The CCD camera was dismounted from the telescope between the 1990 and 1991 series, so a difference in position angle is to be expected. However the same chip was used for all the observations of 1990 and 1991 and the telescope mirrors are of zero-expansion ceramic. Thus the focal length should be the same for both years and also the scale in arcsec/pixel; no significant difference is found here. The present observations also reveal a correlation between position angle and hour angle as found by Jones (1996). The effect is more difficult to measure in these results for 1990 and 1991 because a smaller chip was used than the one on which the discussion of Jones (1996) is based.

\section{The observations}

Table 4 shows that the errors in the present observations arise mostly from errors in the star positions. Near opposition Uranus regresses by roughly one chip width per night so that each night's observations are measured relative to a nearly independent set of stars from the nights before and after. This was confirmed by breaking down the observations into separate nights which revealed significant night to night changes in scale and position angle.

Fundamental Right Ascensions and Declinations are not required for the satellites because their theory can be improved simply from observations of one satellite relative to another. In Table 7 (only available in electronic form at the CDS via anonymous ftp to cdsarc.u-strasbg.fr (130.79.128.5) or via http://cdsweb.ustrasbg.fr/Abstract.html) the satellite positions are presented as standard co-ordinates $(\xi, \eta)$ relative to a reference satellite which is usually Oberon, but otherwise the next satellite observed nearer the planet. In reducing the data the scale and rotation of position angle with hour angle were fixed for the whole series and separate zero points of position angle (J2000.0) were fixed for each year. The constants were found from weighted means over the separate nights. The formulae used were:

$$
\begin{aligned}
& \theta=-88.8647( \pm 26)+0.00527( \pm 106) \tau \\
& \theta=90.2214( \pm 46)+0.00527( \pm 106) \tau \\
& \xi=0^{\prime \prime} 302763( \pm 20)(\Delta x \cos \theta+\Delta y \sin \theta) \\
& \eta=0^{\prime \prime} 302763( \pm 20)(-\Delta x \sin \theta+\Delta y \cos \theta)
\end{aligned}
$$

where $\Delta x, \Delta y$ are measured in pixels on the chip relative to the reference satellite, and $\tau$ is the hour angle in hours. The standard errors are in units of the least significant digit. The standard co-ordinates were corrected for refraction under a pressure of $760 \mathrm{mBar}$; the results are presented in Table 7.

\section{Comparison with Taylor (1998)}

The rms's, and means are given in arcseconds in Table 6 where S.E.M. is the standard error of the mean, $N_{\mathrm{u}}$ is the number of observations used and $N_{\mathrm{r}}$ is the number 
Table 6. Comparison with the numerical integration of Taylor (1998)

\begin{tabular}{|c|c|c|c|c|c|}
\hline Satellite & Rms & Mean & S.E.M. & $N_{\mathrm{u}}$ & $N_{\mathrm{r}}$ \\
\hline All $\xi \& \eta$ & 0..0401 & 0.0009 & 0.0013 & 1017 & 11 \\
\hline All $\xi$ & $0^{\prime \prime} 0423$ & $0^{\prime \prime} 0049$ & 0.'0019 & 511 & 3 \\
\hline All $\eta$ & $0.0^{\prime \prime} 0374$ & $-0.0^{\prime \prime} 0031$ & $0.0^{\prime \prime} 0017$ & 506 & 8 \\
\hline Miranda $\xi$ & 0.0655 & 0.0053 & 0.0061 & 114 & 3 \\
\hline Miranda $\eta$ & $0^{\prime \prime} 0567$ & 0.0045 & 0.0054 & 112 & 5 \\
\hline Ariel $\xi$ & $0^{\prime \prime} 0336$ & $0^{\prime \prime} 0007$ & $0^{\prime \prime} 0029$ & 139 & 0 \\
\hline Ariel $\eta$ & $0^{\prime \prime} 0284$ & $-0^{\prime \prime} 0065$ & $0^{\prime \prime} 0024$ & 139 & 0 \\
\hline Umbriel $\xi$ & 0.'0392 & 0.0086 & $0 .{ }^{\prime \prime} 0034$ & 131 & 0 \\
\hline Umbriel $\eta$ & $0^{\prime \prime} 0330$ & $-0^{\prime \prime} 0034$ & 0.0029 & 130 & 1 \\
\hline Titania $\xi$ & $0^{\prime \prime} 0236$ & 0.0053 & 0.0021 & 127 & 0 \\
\hline Titania $\eta$ & 0.0267 & $-0 .{ }^{\prime \prime} 0058$ & $0.0^{\prime \prime} 0024$ & 125 & 2 \\
\hline
\end{tabular}

of observations rejected. 11 outliers with residuals $>0$ "' 15 were rejected out of 1028 measures.

In deriving these figures no change was made to the initial conditions of Taylor (1998). These values are comparable to the photographic observations of Veiga \& Vieira Martins (1995a) but smaller than the CCD observations of Veiga \& Vieira Martins (1995b). The significance of these differences should not be stressed because Veiga and Vieira Martins compared their observations to Laskar \& Jacobson (1987) where we compared with Taylor (1998).

\section{Conclusions}

The best fit in Table 6, 0.025 , is between Titania and Oberon. We believe this fit is the best because these are the brightest two satellites. However, the typical error of centring a CCD image is 0 .' 012 which should contribute $0^{\prime \prime} 017$ to the separation of two satellites. As all inter-satellite separations have bigger scatters than this we believe that the constants of the integration can be significantly improved on. Further observations are required which we plan to obtain.
Acknowledgements. The Jacobus Kapteyn Telescope is operated on the island of La Palma by the Royal Greenwich Observatory in the Spanish Observatorio del Roque de los Muchachos of the Instituto de Astrofisica de Canarias. The authors thank J.D.H. Pilkington for measuring the plates, R.W. Argyle for help with the photographic observations and A. Steele with the reductions. We thank L.V. Morrison for helpful advice.

\section{References}

Bastian U., Röser S., 1993, PPM Star Catalogue Vol. III, Astronomisches Rechen-Institut Heidelberg

Carter D., Sharan U., Clegg R.E.S., 1995, Isaac Newton Group, Observers' Guide

Harmer C.F.W., Wynne C.G., 1977, MNRAS 177, 25P

Harper D., Murray C.D., Beurle K., Williams I.P., Jones D.H.P., Taylor D.B., Greaves S.C., 1997, A\&AS 121, 65

Jones D.H.P., 1996, IAU Symposium No. 172, Dynamics, Ephemerides and Astrometry in the Solar System, FerrazMello S. and Arlot J.-E. (eds.), p. 431

Laskar J., Jacobson R.A., 1987, A\&A 188, 212

Morrison J., McLean B., 1996, Brief Description of GSC1.2 Space Telescope Science Institute Web Page http://wwwgsss.stsci.edu/gsc/ gsc12/description.html

Pascu D., Seidelmann P.K., Schmidt R.E., Santoro E.J., Hershey J.L., 1987, AJ 93, 963

Reitsema H.J., Smith B.A., Weistrop D.E., 1978, BAAS 10, 585

Secker J., 1995, PASP 107, 496

Taylor D.B., Jones D.H.P., Morrison L.V., Murray C.D., Williams I.P., 1990, A\&A 232, 565

Taylor D.B., 1998, A\&A 330, 362

Veiga C.H., Vieira Martins R., 1995a, A\&AS 111, 387

Veiga C.H., Vieira Martins R., 1995b, A\&AS 113, 557

Vieira Martins R., de Carvalho R.R., Veiga C.H., 1986, Rev. Mex. Astron. Astrofis. 12, 399

Wallace P.T., 1994, ASTROM - Basic Astrometry Program, Starlink User Note 5.14

Zacharias N., 1996, PASP 108, 1135 